\title{
Exercise interventions for older adults with Alzheimer's disease: a systematic review and meta-analysis protocol
}

Julie M. Faieta ${ }^{1,2^{*}+} \mathbb{D}$, Hannes Devos ${ }^{3+}$, Prasanna Vaduvathiriyan ${ }^{4}$, Michele K. York ${ }^{5}$, Kirk I. Erickson ${ }^{6}$, Mark A. Hirsch ${ }^{7}$, Brian G. Downer ${ }^{8}$, Erwin E. H. van Wegen ${ }^{9}$, Diana C. Wong ${ }^{10}$, Elena Philippou ${ }^{11}$, Ahmed Negm ${ }^{12}$, Pedram Ahmadnezhad ${ }^{3}$, Shilpa Krishnan ${ }^{13}$, Melike Kahya ${ }^{3}$, Pallavi Sood ${ }^{14}$ and Patricia C. Heyn ${ }^{15}$

\begin{abstract}
Background: The growing societal and economic impact of Alzheimer's disease (AD) is further compounded by the present lack of disease-modifying interventions. Non-pharmacological intervention approaches, such as exercise, have the potential to be powerful approaches to improve or mitigate the symptoms of AD without added side effects or financial burden associated with drug therapies. Various forms and regiments of exercise (i.e., strength, aerobic, multicomponent) have been reported in the literature; however, conflicting evidence obscures clear interpretation of the value and impact of exercise as an intervention for older adults with AD. The primary objective of this review will be to evaluate the effects of exercise interventions for older adults with AD. In addition, this review will evaluate the evidence quality and synthesize the exercise training prescriptions for proper clinical practice guidelines and recommendations.

Methods: This systematic review and meta-analysis will be carried out by an interdisciplinary collective representing clinical and research stakeholders with diverse expertise related to neurodegenerative diseases and rehabilitation medicine. Literature sources will include the following: Embase, PsychINFO, OVID Medline, and Ovid MEDLINE(R) and Epub Ahead of Print, In-Process \& Other Non-Indexed Citations and Daily. Inclusion criteria are participants with late onset $A D$ and structured exercise interventions with prescribed duration, frequency, and intensity. The primary outcome of this study will center on improved or sustained cognitive functioning. Secondary outcomes will include institutionalization-related outcomes, ability in activities of daily living, mood and emotional well-being, quality of life, morbidity, and mortality. Analysis procedures to include measurement of bias, data synthesis, sensitivity analysis, and assessment of heterogeneity are described in this protocol.
\end{abstract}

(Continued on next page)

\footnotetext{
* Correspondence: Julie.faieta.1@ulaval.ca

†Julie M Faieta and Hannes Devos provided equal contributions and share co-first authorship.

${ }^{1}$ Department of Rehabilitation, Université Laval, Quebec, Canada

${ }^{2}$ Centre interdisciplinaire de recherche en réadaptation et en intégration sociale (CIRRIS), Québec, Canada

Full list of author information is available at the end of the article
}

(c) The Author(s). 2021 Open Access This article is licensed under a Creative Commons Attribution 4.0 International License, which permits use, sharing, adaptation, distribution and reproduction in any medium or format, as long as you give appropriate credit to the original author(s) and the source, provide a link to the Creative Commons licence, and indicate if changes were made. The images or other third party material in this article are included in the article's Creative Commons licence, unless indicated otherwise in a credit line to the material. If material is not included in the article's Creative Commons licence and your intended use is not permitted by statutory regulation or exceeds the permitted use, you will need to obtain permission directly from the copyright holder. To view a copy of this licence, visit http://creativecommons.org/licenses/by/4.0/ The Creative Commons Public Domain Dedication waiver (http://creativecommons.org/publicdomain/zero/1.0/) applies to the data made available in this article, unless otherwise stated in a credit line to the data. 
(Continued from previous page)

Discussion: This review is anticipated to yield clinically meaningful insight on the specific value of exercise for older adults with AD. Improved understanding of diverse exercise intervention approaches and their specific impact on various health- and function-related outcomes is expected to guide clinicians to more frequently and accurately prescribe meaningful interventions for those affected by AD.

Systematic review registration: PROSPERO CRD42020175016.

Keywords: Exercise, Physical activity, Exercise training, Older adults, Dementia, Alzheimer's disease, Mild cognitive impairment, Cognitive function

\section{Background}

Characterized by deficits in memory, cognitive function, and behavior [1], dementia is projected to affect over 48 million individuals globally in 2020, and to increase by over $46 \%$ to a prevalence of 90.3 million by 2040 [2]. Alzheimer's disease (AD) is the most prevalent form of dementia [3], accounting for approximately $60-80 \%$ of the dementia population [4] and affecting over 5.5 million individuals in the USA alone [5]. Alzheimer's disease is a progressive, irreversible brain disorder which impacts memory and thinking, and eventually the ability to carry out basic activities of daily living. Typically, clinical symptoms include declines in episodic memory, behavioral changes, and impaired language and visuospatial functioning [6]. Due to the functional changes associated with the disease, many individuals living with $\mathrm{AD}$ in the community require the help and support of at least one informal caregiver, either a family member or a friend. The Alzheimer's Association reports that the extensive care provided by informal caregivers is valued at over 18 billion dollars annually [4]. Current pharmacotherapy interventions for AD are symptomatic, masking rather than altering the course of the disease [7]. Therefore, non-pharmacological intervention options such as exercise are of great importance in caring for individuals living with the symptoms of AD.

A physically active lifestyle that incorporates exercise, whether through targeted training or recreation, is frequently promoted as a component of a healthy lifestyle. The familiarity of exercise as a wellness concept may lessen its perceived importance. However, exercise should be viewed as a highly impactful tool associated with a myriad of widely accepted benefits such as cardiovascular fitness, improved sleep, and digestive functioning [8]. Specific to healthy aging, research has shown physical activity to delay cognitive decline $[9,10]$. Exercise-based interventions can be practically implemented at a community-wide level to support mental health and wellness in the aging populations via recommended guidelines and community initiatives (e.g., SilverSneakers type programs). In addition to general health and wellness, exercise is a valuable tool across health care settings. Exercise is used across various rehabilitative settings to target skeletal muscle function following an injury or to improve overall functional capacity. Additionally, exercise has shown promise as an effective cognitive enhancement intervention for older adults with cognitive impairments [11-14].

The mechanisms through which exercise may benefit cognitive functioning are likely to be multifactorial. Stillman and colleagues indicate that these mechanisms can be conceptualized on multiple levels to include cellular, molecular, and psychosocial levels [15]. On the molecular and cellular level, sustained routine aerobic exercise in adults reduces inflammation, regulates insulin pathways, suppresses oxidative stress, and contributes to calcium homeostasis [16], all of which may independently or additively influence brain and cognitive outcomes. Furthermore, exercise has been shown to promote various plasticity-related events in the human brain including neurogenesis, synaptogenesis, and angiogenesis. Several randomized trials have demonstrated that exercise alters the size of brain regions in older adults, and these changes might be linked to improvements in cognitive performance $[17,18]$. Yet, few neuroimaging studies have attempted to links these changes to behavior which inevitably leads to the question of the relevance and importance of brain changes detected using neuroimaging methods. Additional consensus of clinical trials of exercise including both neuroimaging and cognitive outcomes are needed.

Mechanisms of exercise effect on cognitive function can also be conceptualized at the psychosocial level. For example, exercise-induced changes in sleep parameters may be mediating cognitive benefits observed with exercise. Another possibility is that the mood-enhancing effects of exercise lead to cognitive improvements. Although these are all possibilities, they are largely speculation at this point given the dearth of literature that tests such hypotheses. One likely scenario is that there are many different pathways that explain how exercise influences cognition and that these pathways might differ as a function of diverse variables, such as, age group, disease state, mode of exercise, duration and volume of exercise, intensity of exercise, or baseline cognitive status.

Despite an increased number of both systematic reviews and meta-analyses published in the last 15 years on the topic of physical activity and dementia, few have attempted 
to evaluate whether physical activity or exercise prescription was effective or in support of current clinical practice recommendations by the American College of Sports Medicine (e.g., Exercise is Medicine (EIM) guideline [19]). Furthermore, some findings indicate that exercise is ineffective in delaying cognitive decline [20]. A recent review of the literature noted inconsistency in the evidence on the value of physical activity for cognitive outcomes in the $\mathrm{AD}$ population, hypothesizing that changes in standardized outcome measures used to assess cognition may be a contributing factor to this apparent incongruity [21]. Studies that do show effectiveness of exercise intervention demonstrate, at best, moderate effects on cognitive function in $\mathrm{AD}$ [22] or simply indicate that evidence is insufficient to draw conclusions [23]. Limited and conflicting evidence thus generates more questions than answers. The longterm impact of effective interventions is unclear, and the type and optimal training parameters (frequency, intensity, and duration) leading to the most effective outcomes have not yet been established. Thus, to address the present lack of understanding, this review aims to develop a more comprehensive analysis of the potential cognitive functioning outcomes related to exercise in individuals with AD. This review will not only evaluate the evidence, its quality, and bias, but it will also synthesize the intervention doses and prescriptions for proper clinical practice guidelines and recommendations. The need to evaluate specific exercise dosages is highlighted by Guitar et al. where the authors concluded that "Future studies should explore the benefits of the American College of Sports Medicine recommended 150 min of physical exercise per week with select measures of executive function [24], p. 159."

As research presses toward a cure for $\mathrm{AD}$, it is imperative to critically assess the existing, feasible intervention approaches in order to support the quality of life for those already experiencing AD-related decline. Exercise has the potential to mitigate the physical and cognitive decline associated with $\mathrm{AD}$ progression, but inconsistent support for exercise as an intervention for cognitive function necessitates critical assessment of the literature. This systematic review protocol details the aims, methods, and anticipated findings of an upcoming review on exercise interventions for older adults with Alzheimer's disease. The primary objective of the present systematic review and meta-analysis is to assess the effects of structured exercise regiments on cognitive function in older adults with AD. Secondary objectives of this review include assessing the effects of structured exercise regiments on institutionalization, activities of daily living, emotional well-being, quality of life, morbidity, and mortality.

\section{Methods}

This protocol has been registered under the PROSPERO database (registration number CRD42020175016). We will follow the standards for best practice in transparent, reproducible, and ethical reporting of systematic reviews per the Preferred Reporting Items for Systematic Reviews and Meta-Analyses Protocols (PRISMA-P) statement (see Additional File 1) [25].

\section{Eligibility criteria}

For the purposes of this review, only randomized controlled trials will be considered. We will evaluate and include studies according to the following criteria: participants, interventions, outcome(s) of interest, and study design. Participant inclusion criteria will include at least one sample group with Alzheimer's disease, as described by individual study authors. We will include studies with additional diagnostic or caregiver groups, but inclusion of participants with $\mathrm{AD}$ and outcomes specific to those with this condition will be considered an inclusion criterion. To be considered eligible for inclusion, studies must also contain one or more exercise-based intervention. To be able to evaluate the exercise prescriptions (dose, mode, type), we will consider only structured and planned exercise interventions of prescribed frequency, dose, and/or intensity as specified by study authors. For the purposes of this review, we will use the following definition of exercise, sourced from the Physical Activity Guidelines Advisory Committee "physical activity that is planned, structured, repetitive, and designed to improve or maintain physical fitness, physical performance, or health [26]. Exercise, like physical activity, encompasses all intensities [27], p. C-3.". We will use the term exercise synonymously with structured physical activity throughout this review.

Based on this definition, we will include both resistance and aerobic exercises. Examples include, but are not limited to, walking, swimming, weight training, and use of exercise equipment in a home or gym setting that are reported with prescribed time, frequency, and/or intensity (see examples in Table 1).

We will classify comparative doses of exercise as uniform with the primary intervention group (for example, strength training compared to alternative dose of strength training) or variant (for example, strength training, compared to non-strength training forms of exercise). Only control (non-exercise groups) and comparative dose groups will be considered.

Table 1 Example intervention parameters

\begin{tabular}{|c|c|c|c|}
\hline Activity & Frequency & Dose & Intensity \\
\hline Walking & 30 min, $2 \times$ per week & 10 weeks & Moderate pace \\
\hline Waltz dancing & $1 \mathrm{~h}, 1 \times$ per week & 7 weeks & Intermediate dance \\
\hline Pilates & 45 min, $3 \times$ per week & 5 weeks & Low intensity \\
\hline Pickle ball & 3-h sets, $2 \times$ per month & 6 months & $5 \mathrm{METS}$ \\
\hline
\end{tabular}




\section{Outcomes}

The primary outcomes of our review will be cognitive improvement and/or sustained cognitive functioning in older adults with $\mathrm{AD}$. While $\mathrm{AD}$ impacts many areas of behavioral and psychosocial functioning, this review seeks to provide an in-depth and comprehensive investigation on a narrow area deeply affected by $\mathrm{AD}$, specifically, the impact of exercise on cognitive ability within AD populations. Therefore, cognitive function will be considered our primary area of focus. We will also explore specific cognitive domains (i.e., memory, language, attention and executive function, and visuospatial function).

Secondary outcomes will be explored through subanalyses pending review results. While our primary area of concern is cognitive function, we will also consider additional outcomes that are typically associated with $\mathrm{AD}$ disease progression to better understand the global impact of exercise on AD. Anticipated secondary outcomes of interest include the impact of exercise on:

- Institutionalization-related outcomes (such as caregiver burden, level of assistance for activities of daily living, time to and rate of institutionalization);

- Ability in functional activities and activities of daily living;

- Mood and emotional well-being;

- Caregiver outcomes such as health, depression, participation, and function;

- Quality of life-related outcomes (such as social participation);

- Morbidity and overall mortality.

Both clinically important and statistically significant differences will be included as appropriate. Finally, we will report applicable incidence of adverse events such as injury or pain with exercise participation.

\section{Information sources and search strategy}

We will conduct comprehensive literature searches in the following databases (from their inception onwards):

- Embase.com, 1947-present

- Ovid Medline, Ovid MEDLINE(R) and Epub Ahead of Print, In-Process \& Other Non-Indexed Citations, Daily and Versions(R), 1946 to present.

- PsycINFO (Ebsco interface), 1734-present

A combination of keywords and controlled vocabularies (when available in the databases) are used for conducting the searches. Search techniques such as adjacency search and truncating are used in order to increase the sensitivity in results. Search results will be exported, and duplicates will be removed using EndNote [version X9.2]. The draft search strategies are provided in Additional file 2.

\section{Screening and selection procedure}

All titles and abstracts will be reviewed by two independent reviewers to determine appropriateness to the purposes of this review. A third reviewer will resolve any conflicts through consensus. Similarly, two authors will review full texts independently and compare these against predefined eligibility criteria. If necessary, review authors will contact article authors to confirm the article's appropriateness for inclusion in this review. All review authors hold expertise relevant to the review subject matter. A third review author will resolve conflicts between independent review authors regarding inclusion/exclusion of articles. An excluded study list will record articles that did not meet inclusion criteria, but that may provide information of interest to readers.

\section{Data collection}

We will extract data of interest from all included studies using revised versions of a previously piloted data extraction form. All review screening, bias assessment, and data extraction will be managed on the Covidence review management software. Specifically, we will extract variables outlined in Table 2 [28].

\section{Risk of bias in individual studies}

We will use the Risk of Bias 2 Tool (RoB 2) [29] to assess the risk of bias in all included articles at the study level. At least two review authors will assess risk of bias across the five RoB 2 domains, specifically bias related to randomization, deviation from intervention, missing data, measurement, and result selection [29]. A third review author will resolve any conflicts regarding risk of bias considerations. Finally, review authors will provide a rationale for all choices regarding risk of bias. A stratified analysis will be used to assess and report the levels of bias identified across included studies.

\section{Data synthesis}

\section{Qualitative synthesis}

Abstracted and synthesized information will be organized in a tabular fashion. Summary tables will be used to explore the primary and secondary outcomes of interest extracted from included studies. The narrative summary in the tables will include the following: author, year of publication, country where the study was conducted, study objectives, population studied, outcome measures used, risk of bias, and major findings (see Table 1).

\section{Quantitative synthesis}

If two or more of the included studies reported the effect of the exercise intervention on a primary or secondary outcome, we will pool the eligible studies using a meta-analysis approach. Due to the expected 
Table 2 Data extraction variables

\begin{tabular}{l} 
Variable \\
\hline Study inclusion/exclusion \\
Design and setting \\
Dates \\
$N$, number of groups, and \\
dropout rates \\
Participant demographics
\end{tabular}

Included caregiver or caretaker

Recruitment approach

Adherence reported according to For example, supervised, how intervention was recorded unsupervised, objective monitors, etc.

Follow-up assessment

Outcomes

Reported challenges and conflicts

Bias assessment variables

Outcome description

Other outcome factors

Results: included participant group sizes, summary of results

$$
\begin{aligned}
& \text { To include cognitive, emotional, } \\
& \text { functional, motor, physiological, } \\
& \text { social, or quality of life }
\end{aligned}
$$

This will include baseline cognitive status and activity or fitness level, diagnosis, disease severity, comorbidities, Apo-E status, age, sex nationality and/or ethnicity, socio economic status, and education

To include randomization method, randomization concealment, blinding, method of reporting outcomes, missing information, and other relevant variables

To include scale range; minimal detectable change; and/or minimal clinically important difference; incidence of dementia; time to conversion; changes in health, cognitive, or quality of life variables; and changes in dementia related biomarkers

To include time to follow-up in study methodology, scale range, minimal detectable change, and/or minimal clinically important difference

For example, means, SD, and statistical changes

Physical activity/exercise treatment prescription data

Physical activity/exercise modality

For example, type, group, peer/ coach, and additional motivators

Treatment minutes/session

Treatment duration

Total number of weeks

Supervised or unsupervised

treatment paradigm

Harm or side effects

Drug or secondary health condition interaction

heterogeneity of the included studies, we will use the inverse-variance random-effect model.

Revman version 5 will be used to pool estimates of the effect sizes on primary and secondary outcomes. Summary of pooled estimates will be also presented graphically in terms of forest plots.

\section{Measures of treatment effect}

For dichotomous outcomes, we will calculate the odds ratio with a 95\% confidence interval. For continuous outcomes, we will calculate the mean difference with a 95\% confidence interval. We will use the standardized mean difference with a $95 \%$ confidence interval if the included trials use different scales for a continuous outcome. Hazard ratio will measure time-to-conversion to dementia data. We will use intention-to-treat data, and, if not available, we will only use the reported completers' data in the analysis. The between-study heterogeneity will be measured using the chi-squared test. For chi-squared values with $P<0.1$, heterogeneity will be considered to be significantly high. The $I^{2}$ will be used to assess the inconsistency between the pooled studies [30]. The heterogeneity is determined as low, medium, or high when the $I^{2}$ test values are $25 \%, 50 \%$, or $75 \%$, respectively [30]. The $I^{2}$ of $<70 \%$ will be considered to be acceptable for pooling the data across the studies [30]. Any comparison with high heterogeneity will be explored by a subgroup analysis.

\section{Subgroup analyses}

If sufficient studies are available (3 or more studies), we will perform subgroup analyses based on characteristics thought to modify treatment effects or possible sources of heterogeneity between studies such as age, gender, educational level, comorbidity, patient groups, types of intervention, or types of study. Our a priori hypothesis will be that older, lower educational level, and more comorbidity subgroups may show less improvement in the primary and secondary outcomes. We will also stratify our results by additional study method variables as appropriate to include supervised (exercise observed by clinical or exercise professional) versus unsupervised protocols, high versus low risk of bias, measurement through fitness versus heart rate, quality of the cognitive assessment used, and adherence over $80 \%$.

\section{Sensitivity analysis}

We will use sensitivity analyses to assess the appropriateness of including various modes of exercise, such as aerobic and resistance training intervention protocols. In addition, we will use sensitivity analyses to assess whether or not the inclusion of studies with missing outcome data of varying degrees meaningfully impacts the results of the meta-analysis.

\section{Meta-bias and quality of evidence}

For each comparison with 10 or more studies, we will visually assess publication bias and small-study effects 
using funnel plots (using study's effect estimates for the primary outcomes against their standard errors). We will utilize GRADE approach to determine the quality of evidence of included articles, designating all articles to one of four levels of evidence according to their study and other variables which have the potential to impact the quality of evidence (such as indirect evidence, specific types of bias, and effect size) [28].

\section{Discussion}

This review protocol builds upon a growing body of literature to critically assess high-quality evidence in order to determine the conclusive impact of exercise on cognitive function. Declines in cognitive function, including memory, visuospatial skills, language skills, and executive function are hallmark in $\mathrm{AD}$ [6]. These cognitive changes are often paired with declines in activities of daily living and the ability to participate in meaningful tasks and social relationships. To address these deficits, pharmacotherapies such as acetylcholinesterase inhibitors and a N-methyl-D-aspartate (NMDA) agonist [7] are typically prescribed to help compensate for the effects of the disease. The benefits of pharmacotherapy can, unfortunately, be paired with unwanted side effects. Furthermore, these medications do not inhibit the progression of $\mathrm{AD}$. The stress and negative mental health outcomes experienced by AD caregivers [31-33] further emphasize the importance of identifying and implementing effectual interventions for this population. Improved cognitive ability, subsequently enabling improved functional capacity, may lessen the responsibilities and challenges that informal caregivers face as they support their loved one with $\mathrm{AD}$. A holistic and client-centered approach to caring for an individual with $\mathrm{AD}$ must then be appropriately informed of the value of supplementing pharmacotherapy with non-pharmacological interventions such as exercise prescriptions.

In addition to the direct physiological benefits of exercise, the accessibility and socioeconomic considerations of this intervention approach warrant its further consideration. Structured physical activity, if appropriately designed, can accommodate individuals with $\mathrm{AD}$ across diverse lifestyles and contexts. The recent outbreak of COVID-19 has brought attention to the importance of health care that can be prescribed remotely and carried out in one's home. Exercise meets both of these criteria and is a highly malleable intervention, feasibly modifiable for context and safety during periods of social isolation or distancing.

We anticipate the outcomes of this review will indicate that structured exercise yields statistically and clinically significant improvements in health and quality of life factors. The effects of specific exercise protocols on various cognitive domains may imply the importance of certain volumes and intensities of exercise to address specific deficits. The results of this review are intended to guide clinical prescription and uptake of exercise protocols among the AD population as a targeted and effective intervention approach. In addition, the results of this review will likely hold implications for the impact of exercise on cognitive outcomes in additional neurodegenerative and age-related conditions. Once the review and meta-analysis is complete, results will be disseminated through both scientific, peer-reviewed journal article(s) and conference presentation. Any amendments made to this protocol when conducting the study will be outlined in PROSPERO and reported in the final manuscript.

\section{Potential limitations}

The potential limitations of this systematic review include lack of clear and transparent reporting within included studies (for example, inclusion or exclusion criteria; cognitive level among sample participants; lack of consideration for gender, race, and socioeconomic status). A second potential limitation is the failure to include underrepresented minorities within study sample populations. Dependent on the reporting practices of the included studies, we also anticipate the potential for high heterogeneity due to lack of methodological rigor, for example, lack of blinding, lack of well-trained exercise specialist personnel, and failure to report if other motivational and engagement approaches were used in conjunction with the exercise treatment (e.g., music, group socialization, technology-based support).

There are several operational issues that we anticipate for this study. Firstly, lack of common data elements across studies may prove challenging in data extraction processes. A second potential issue is the likelihood that selection bias toward a sample of individuals with a relatively high literacy and socioeconomic status will limit generalizability of results to the general AD population. Finally, lack of data sharing and open access within science practices may provide additional challenges to the processes of this study.

This review is designed to assess and provide clinically relevant information regarding the impact of exercise on cognitive outcomes for those living with $\mathrm{AD}$. Low cost, non-pharmacological and non-invasive interventions, such as exercise, are crucial considering the aging population and growing prevalence of AD. Interventions that can be implemented early in the disease process and in a person's individual community context have the potential to significantly impact public health. Therefore, it is imperative to rigorously scrutinize, then purposefully implement the most effective interventions to help mitigate physical and cognitive decline associated with a diagnosis of AD. In sum, we anticipate that this review will yield clinically and socially meaningful insight into the impact of exercise on cognitive function in the AD population. 


\section{Supplementary Information}

The online version contains supplementary material available at https://doi. org/10.1186/s13643-020-01555-8.

Additional file 1. PRISMA-P 2015 Checklist.

Additional file 2. Search Strategies.

\section{Abbreviation}

AD: Alzheimer's disease; ElM: Exercise is Medicine; NMDA: N-methyl-Daspartate; RoB 2: Risk of Bias 2 Tool; SD: Standard Deviation

\section{Acknowledgements}

Not applicable.

\section{Authors' contributions}

$\mathrm{JF}, \mathrm{HD}$, and $\mathrm{PH}$ have contributed to the conception and design of this protocol. JF and HD provided equal contributions and share co-first authorship. $\mathrm{DH}, \mathrm{LH}$, and PV contributed to the development of the search component of this protocol. MY, KE, MH, BG, EvW, DW, EP, AN, PA, SK, MK, and PS have assisted in the draft and review of this protocol. Several authors have specialized roles as statistical advisors (AN, PA, and SK) and psychology consults (KE and MY). All authors have read and approved this manuscript

\section{Funding}

None.

\section{Availability of data and materials}

The data used in this study will be extracted from published literature identified through Ovid MEDLINE(R) and Epub Ahead of Print, In-Process \& Other Non-Indexed Citations and Daily.

\section{Ethics approval and consent to participate} Not applicable.

\section{Consent for publication}

Not applicable. Due to the study design, no human data was collected; therefore, consent for publication was not attained from any persons.

\section{Competing interests}

$\mathrm{MH}$ declares the following interests: MAH, grant funding from Merz Neurosciences, Dutch Brain Foundation, Dutch Parkinson Patient Association. Editorial Board, Parkinsonism and Related Disorders, speaker honoraria from International Association of Parkinsonism and Related Disorders. Nonfinancial: American Congress of Rehabilitation Medicine-Neurodegenerative Diseases Networking Group. All remaining authors declare that they have no competing interests.

\section{Author details}

'Department of Rehabilitation, Université Laval, Quebec, Canada. ${ }^{2}$ Centre interdisciplinaire de recherche en réadaptation et en intégration sociale (CIRRIS), Québec, Canada. ${ }^{3}$ Department of Physical Therapy and Rehabilitation Science, University of Kansas Medical Center, Kansas City, USA. ${ }^{4}$ Dykes library, University of Kansas Medical Center, Kansas City, USA. ${ }^{5}$ Neurology, Baylor College of Medicine, Houston, USA. ${ }^{6}$ Psychology, University of Pittsburgh, Pittsburgh, USA. ${ }^{7}$ Physical Medicine and Rehabilitation, Carolinas Medical Center/Carolinas Rehabilitation, Charlotte, USA. ${ }^{8}$ Division of Rehabilitation Sciences, School of Health Professions, University of Texas Medical Branch, Galveston, USA. ${ }^{9}$ Department of Rehabilitation Medicine, Amsterdam Neurosciences, Amsterdam Movement Sciences, Amsterdam, Netherlands. ${ }^{10}$ Occupational Therapy, Rehabilitation Department, New York University, New York, USA. " ${ }^{11}$ Life and Health Sciences, University of Nicosia, Nicosia, Cyprus. ${ }^{12}$ Rehabilitation Medicine, University of Alberta, Edmonton, Canada. ${ }^{13}$ Rehabilitation Medicine, Division of Physical Therapy, Emory University, Atlanta, USA. ${ }^{14}$ Rehabilitation Science, University of Florida, Gainesville, USA. ${ }^{15}$ Physical Medicine and Rehabilitation, University of Colorado Anschutz Medical Campus, Aurora, USA.
Received: 4 May 2020 Accepted: 6 December 2020

Published online: 04 January 2021

\section{References}

1. Chapman DP, Williams SM, Strine TW, Anda RF, Moore MJ. Dementia and its implications for public health. Prev Chronic Dis. 2006;3(2):A34.

2. Prince M, Bryce R, Albanese E, Wimo A, Ribeiro W, Ferri CP. The global prevalence of dementia: a systematic review and metaanalysis. Alzheimers Dement. 2013:9(1):63-75.e2. https://doi.org/10.1016/j.jalz2012.11.007.

3. Barker WW, Luis CA, Kashuba A, et al. Relative frequencies of Alzheimer disease, Lewy body, vascular and frontotemporal dementia, and hippocampal sclerosis in the State of Florida Brain Bank. Alzheimer Dis Assoc Disord. 2002;16(4):203-12.

4. Gaugler J, James B, Johnson T, Marin A, Weuve J. 2019 Alzheimer's disease facts and figures. Alzheimers Dement. 2019;15(3):321-87.

5. Hebert LE, Weuve J, Scherr PA, Evans DA. Alzheimer disease in the United States (2010-2050) estimated using the 2010 census. Neurology. 2013; 80(19):1778-83. https://doi.org/10.1212/WNL.0b013e31828726f5.

6. McKhann GM, Knopman DS, Chertkow H, et al. The diagnosis of dementia due to Alzheimer's disease: recommendations from the National Institute on Aging-Alzheimer's Association workgroups on diagnostic guidelines for Alzheimer's disease. Alzheimers Dement. 2011;7(3):263.

7. Massoud F, Gauthier S. Update on the pharmacological treatment of Alzheimer's disease. Curr Neuropharmacol. 2010;8(1):69-80. https://doi.org/ 10.2174/157015910790909520.

8. Benefits of exercise. https://medlineplus.gov/benefitsofexercise.html. Accessed 27 Feb 2020

9. ten Brinke LF, Bolandzadeh N, Nagamatsu LS, et al. Aerobic exercise increases hippocampal volume in older women with probable mild cognitive impairment: a 6-month randomised controlled trial. Br J Sports Med. 2015;49(4):248-54. https://doi.org/10.1136/bjsports-2013-093184.

10. Yu F, Bronas UG, Konety $\mathrm{S}$, et al. Effects of aerobic exercise on cognition and hippocampal volume in Alzheimer's disease: study protocol of a randomized controlled trial (The FIT-AD trial). Trials. 2014;15:394. https://doi. org/10.1186/1745-6215-15-394.

11. Colcombe S, Kramer AF. Fitness effects on the cognitive function of older adults: a meta-analytic study. Psychol Sci. 2003;14(2):125-30. https://doi.org/ 10.1111/1467-9280.t01-1-01430.

12. Heyn $P$. The effect of a multisensory exercise program on engagement behavior, and selected physiological indexes in persons with dementia. Am J Alzheimers Dis Other Demen. 2003:18(4):247-51. https://doi.org/10.1177/ 153331750301800409

13. Heyn P, Abreu BC, Ottenbacher KJ. The effects of exercise training on elderly persons with cognitive impairment and dementia: a meta-analysis. Arch Phys Med Rehabil. 2004;85(10):1694-704. https://doi.org/10.1016/j. apmr.2004.03.019.

14. Heyn PC, Johnson KE, Kramer AF. Endurance and strength training outcomes on cognitively impaired and cognitively intact older adults: a meta-analysis. J Nutr Health Aging. 2008;12(6):401-9. https://doi.org/10. 1007/bf02982674

15. Stillman CM, Cohen J, Lehman ME, Erickson KI. Mediators of physical activity on neurocognitive function: a review at multiple levels of analysis. Front Hum Neurosci. 2016;10:626. https://doi.org/10.3389/fnhum.2016.00626.

16. Dishman RK, Berthoud H-R, Booth FW, et al. Neurobiology of exercise. Obesity (Silver Spring). 2006;14(3):345-56. https://doi.org/10.1038/oby. 2006.46

17. Zhu Y, Wu H, Qi M, et al. Effects of a specially designed aerobic dance routine on mild cognitive impairment. Clin Interv Aging. 2018;13:1691-700. https://doi.org/10.2147/CIA.S163067.

18. Hong S-G, Kim J-H, Jun T-W. Effects of 12-week resistance exercise on electroencephalogram patterns and cognitive function in the elderly with mild cognitive impairment: a randomized controlled trial. Clin I Sport Med. 2018;28(6):500-8. https://doi.org/10.1097/JSM.0000000000000476.

19. American College of Sports Medicine. Exercise is medicine: healthcare providers' action guide. In: Exercise is Medicine. Indianopolis: American College of Sports Medicine; 2014. https://www.exerciseismedicine.org/ support_page.php/health-care-providers/. Accessed 2 Nov 2020.

20. Sabia S, Dugravot A, Dartigues J-F, et al. Physical activity, cognitive decline, and risk of dementia: 28 year follow-up of Whitehall II cohort study. BMJ. 2017;357. https://doi.org/10.1136/bmj.j2709. 
21. Cui MY, Lin Y, Sheng JY, Zhang X, Cui RJ. Exercise intervention associated with cognitive improvement in Alzheimer's disease. Neural Plast. 2018;2018: 9234105. https://doi.org/10.1155/2018/9234105.

22. Hindin SB, Zelinski EM. Extended practice and aerobic exercise interventions benefit untrained cognitive outcomes in older adults: a meta-analysis. J Am Geriatr Soc. 2012;60(1):136-41. https://doi.org/10.1111/j.1532-5415. 2011.03761.x

23. Brasure $M$, Desai $P$, Davila $H$, et al. Physical activity interventions in preventing cognitive decline and Alzheimer-type dementia: a systematic review. Ann Intern Med. 2018;168(1):30-8. https://doi.org/10.7326/M17-1528.

24. Guitar NA, Connelly DM, Nagamatsu LS, Orange JB, Muir-Hunter SW. The effects of physical exercise on executive function in community-dwelling older adults living with Alzheimer's-type dementia: a systematic review. Ageing Res Rev. 2018;47:159-67. https://doi.org/10.1016/j.arr.2018.07.009.

25. Moher D, Shamseer L, Clarke M, et al. Preferred reporting items for systematic review and meta-analysis protocols (PRISMA-P) 2015 statement. Syst Rev. 2015:4(1):1. https://doi.org/10.1186/2046-4053-4-1.

26. Caspersen CJ, Powell KE, Christenson GM. Physical activity, exercise, and physical fitness: definitions and distinctions for health-related research. Public Health Rep. 1985;100(2):126-31.

27. 2018 Physical Activity Guidelines Advisory Committee. US Department of Health and Human Services. Published online 2018:779.

28. Cochrane Handbook for Systematic Reviews of Interventions. https:// training.cochrane.org/handbook/archive/v5.1/. Accessed 27 Feb 2020.

29. Sterne JAC, Savović J, Page MJ, et al. RoB 2: a revised tool for assessing risk of bias in randomised trials. BMJ. 2019;366:14898. https://doi.org/10.1136/ bmj.14898.

30. Higgins JPT, Thompson SG. Quantifying heterogeneity in a meta-analysis. Stat Med. 2002;21(11):1539-58. https://doi.org/10.1002/sim.1186.

31. Brodaty H, Woodward M, Boundy K, Ames D, Balshaw R. Prevalence and predictors of burden in caregivers of people with dementia. Am J Geriatr Psychiatry. 2014;22(8):756-65. https://doi.org/10.1016/j.jagp.2013.05.004.

32. Park M, Sung M, Kim SK, Kim S, Lee DY. Multidimensional determinants of family caregiver burden in Alzheimer's disease. Int Psychogeriatr. 2015;27(8): 1355-64. https://doi.org/10.1017/S1041610215000460.

33. Liu S, Li C, Shi Z, et al. Caregiver burden and prevalence of depression, anxiety and sleep disturbances in Alzheimer's disease caregivers in China. J Clin Nurs. 2017;26(9-10):1291-300. https://doi.org/10.1111/jocn.13601.

\section{Publisher's Note}

Springer Nature remains neutral with regard to jurisdictional claims in published maps and institutional affiliations.

Ready to submit your research? Choose BMC and benefit from:

- fast, convenient online submission

- thorough peer review by experienced researchers in your field

- rapid publication on acceptance

- support for research data, including large and complex data types

- gold Open Access which fosters wider collaboration and increased citations

- maximum visibility for your research: over $100 \mathrm{M}$ website views per year

At BMC, research is always in progress.

Learn more biomedcentral.com/submissions 